\title{
Ice-ocean dynamics and mechanics: a summary of the papers
}

\author{
KOLUMBAN HutTeR \\ Institut für Mechanik, Technische Hochschule Darmstadt, \\ Hochschulstrasse 1, D-6100 Darmstadt, Germany
}

\begin{abstract}
A subjective review and summary of the key ideas presented at the conference is given, with occasional indications as to which scientific steps might resolve specific queries that arose from the work. The intention is to encourage closer reading of the papers.
\end{abstract}

\section{SCOPE}

Participants might have wondered whether the conference topic was Ice-ocean dynamics and mechanics, and thus dealt with modelling ice-ocean interaction processes, or Ice-ocean dynamics and Mechanics, a dual feature of the physical processes of ice interacting with ocean waters and, separated from this, mechanics of ice with all its engineering and geophysical aspects. It was the latter, as one was able to find out quickly.

\section{SEA-ICE DYNAMICS, SEA-ICE MECHANICS}

The two topics cannot be decoupled, as sea-ice dispersion under atmospheric and oceanic driving forces (a problem of sea-ice dynamics) depends on the formulation of the constitutive response of the dispersive stress (a problem of mechanics).

The conference topic was adequately started with Hoeber's presentation on "Sea-ice dynamics in the Weddell Sea in winter". On the basis of observed ice-drift motions of a few buoys, wind stress and ocean stress, and an assumed linear viscous constitutive relationship for the dispersive ice stress (due to Hibler), time-series of shear and bulk viscosities $\eta$ and $\zeta$ were determined. It was found that their values were fluctuating, with nonnegligible episodes of strong negative shear and bulk viscosities. Of course, such negative values are questionable on thermodynamic grounds; it was, however, pointed out by Hoeber in the discussion that negative viscosities would also occur in atmospheric and oceanic applications. It is the writer's personal opinion that such counter-gradient behaviour (actually, with negative viscosity, the flux term is with and not against the gradient, but we adhere to the common term "counter-gradient") is unphysical and requires replacement of the constitutive postulate. Non-linear behaviour (viscosities, for example, depend on the second stress invariant, perhaps in the form of a power law) could provide a remedy. More effective, however, would most likely be higher order closure conditions, as employed in bulk theories of turbu- a)

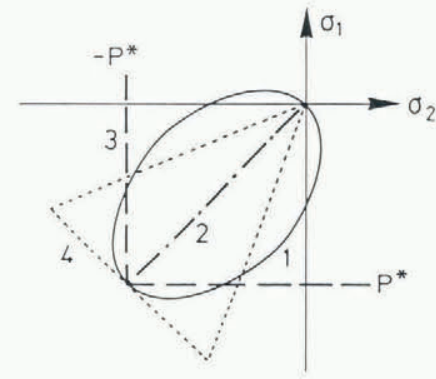

b)

Fig. 1. Various yield surfaces characterizing sea-ice yield under compaction: (a) elliptical (1), cavitation fluid (2), square (3) and Mohr-Coulomb (4) yield surfaces used in a seasonal sea-ice model; (b) typical yield surface as obtained from compacting an assemblage of discs. The strain rates at yield are only nearly orthogonal to the yield surface and the dashed position of the yield surface is uncertain. However, the model does not predict intersection of the yield surface with the principal stress axes. The inset shows the strain rates to which the boundaries of the assembly were subjected.

lent motion. A statistical theory of colliding disks may provide some guidelines as to the possible forms of such closure conditions.

The paper of Ip and others studies the effect of rheology on the evolution of sea ice under seasonal driving forces; only ideally rigid plastic behaviour is analyzed with (Fig. 1a) elliptical (1), square (3), cavitation fluid(2) and Mohr-Coulomb (4) yield surfaces, the first three exhibiting the orthogonality condition of the associated flow rule. All four rheologies produce reasonable ice-velocity patterns, but ice-thickness build-up and iceedge evolution in the two-year simulations are similar for the elliptical and square cases and better for those than for the other two cases. It appears that the optimal 
rheology cannot be singled out; this raises the question as to whether other (non-linear) viscous stress responses would yield equally favourable results at possibly less computing cost.

Perhaps a partial answer to this latter question is provided by Hopkins and Hibler's numerical simulations of a compact, convergent system of ice floes. A large set of contacting disks, with a random distribution of diameters, is confined to a rectangular control area whose sides are subject to prescribed strain rate, from isotropic convergence through pure shear to divergence (Fig. 1b). Interaction forces are viscous plastic, with a prescribed yield, and sliding forces are Mohr-Coulomb. The average stress is computed through the contact forces and its value at yield is determined (this is the value of the stress-tensor when any one of the inner forces reaches yield). A yield surface is typically obtained (Fig. 1b), but the normality condition (the plastic flow rule) is only approximately found to hold. Computations with net divergence are deformation-dependent, but they show that the yield surface does not cross the axes of principal stresses, as for the elliptical yield surface, but rather approaches the origin smoothly from one quadrant only, as is the case for the tear drop and the lenticular yield curves. This result is significant because the modelled material sustains uniaxial compression this way and is thus, for example, not capable of forming arches. In any case, the existence of a yield surface hinges on the assumption of the existence of yield of the normal forces between contacting discs. Yield is thus incorporated $a b$ initio.

Apart from Hopkins and Hibler, the above works all concentrate on the simplest possible sea-ice model, treating it dynamically as a two-dimensional, single component continuum with two mass balances for the ice and lead distributions, respectively. A full multi-layer model being excessively complicated, Flato and Hibler introduced an in-between model where sea ice was treated with one two-dimensional momentum balance and three mass balances for the leads, $A$, level ice, $h_{1}$, and ridged ice, $h_{\mathrm{r}}$, accounting for thermodynamic growth, $G_{\alpha}$, volume transfer, $S_{\alpha}$, and open- water creation, $Q_{\alpha}$, through appropriate production terms,

$$
\begin{aligned}
\frac{\mathrm{d} h_{\mathrm{r}}}{\mathrm{d} t} & =G_{h \mathrm{r}}+S_{\mathrm{r}}+Q_{\mathrm{A}} h_{\mathrm{l}}, \\
\frac{\mathrm{d} h_{1}}{\mathrm{~d} t} & =G_{h \mathrm{l}}+S_{\mathrm{r}}-Q_{\mathrm{A}} h_{\mathrm{l}}, \text { and } \\
\frac{\mathrm{d} A}{\mathrm{~d} t} & =G_{\mathrm{A}}-Q_{\mathrm{A}},
\end{aligned}
$$

with suitable postulates for the various production and sink terms (Fig. 2a). Three-year simulations of the Arctic Basin and the Greenland Sea reveal that this model exhibits more intense ridging near the coasts, in qualitative agreement with observations. Sensitivity studies illustrate the dependence of calculated ridged-ice fraction on pack-ice strength and the requirement for energetically consistent deformation. The paper does not seem to provide inferences conclusive enough to decide whether this model, the simpler two-level or the more complex multi-layer models should be pursued in future work. a)
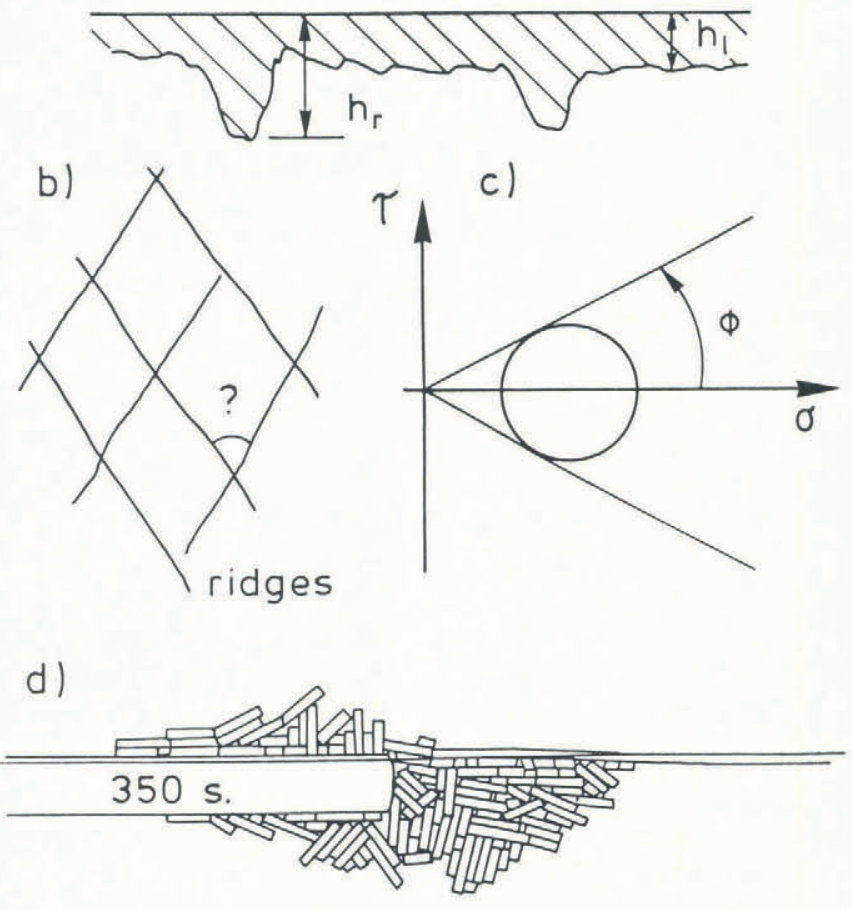

e)

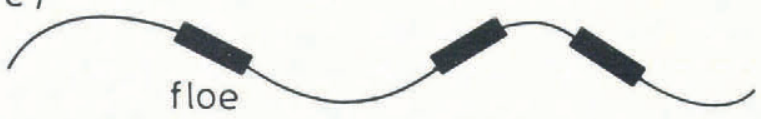

Fig. 2. (a) Definition of $h_{r}$, and $h_{l}$ for ridge and level ice. (b) Regular arrangement of ridges whose intersection angle can be related to $\phi$. (c) Mohr-Coulomb yield surface with constant internal angle of friction $\phi$. Ridge formation from a thin sheet of lead ice. (e) Ice floes riding on a wavy surface.

Whereas the above analyses touch upon the fundamentals of sea-ice mechanics, there are a number of studies dealing with sea-ice dynamics within the coupled atmosphere-ice-ocean systems. The role of the ocean circulation and of seasonal and interannual ice-edge variations is studied by Hibler and associates. Zhang and Hibler give an account of how grid resolution affects icemargin position, Ries and Hibler use a fine resolution of the Arctic Ocean to predict interannual characteristics and Hibler and Ranelli follow a coupled ice-ocean model through 50 annual cycles. These studies show (naturally) that finer grid resolution allows better prediction of the baroclinic ocean circulation, that vertical diffusion processes are important and, perhaps most significant, that precipitation ought to be properly included in interannual sea-ice modelling because it affects energy balance considerably and, consequently, ice-margin positions. Warn-Varnas and others, on the other hand, use a Hibler thermodynamic ice model in conjunction with a three-layer upper-ocean and a one-layer interiorocean model to predict atmospherically driven seasonal ice-ocean structure.

There are also analyses that could be classified as sea- 
ice mechanics, but are not following the main thrust of Hibler-type formulations. Pritchard derives, from the sea-ice momentum balance, a mechanical energy budget (the well known balance of kinetic energy) and demonstrates by using wind, ice motion and current measurements in the Chukchi Sea that order-of-magnitude estimates of the various terms can be more easily obtained with this kinetic energy balance than with the original momentum balance. Erlingsson, on the other hand, supposes that sea ice may be modelled as a plastic material following a Mohr-Coulomb yield criterion (Fig. 2b and c) and explains (by using the method of characteristics and restricting himself to cohesionless behaviour and a constant angle of internal friction, $\phi$ why typical features seen on photographs and satellite images (ridges, diamond-like patterns in the deformation field, regular arrangement of leads) align themselves the way they do. Ridges may, for instance, be viewed as field singularities (corresponding to mathematical characteristics) and their intersecting angles can be related to the internal angle of friction of the Mohr-Coulomb yield criterion.

A refreshing presentation was a video film by Hopkins on ridge formation from a thin sheet of lead ice (Fig. 2d), accompanied by music from Ravel's Bolero. The movie was inspirational and prompted Weeks to remark that he would prefer to see this music accompanied by a movie with Bo Derek, the association obviously being Bo-Lero. Hopkins and Hibler have produced a paper "On the ridging of a thin sheet of lead ice" from this movie, from which we can learn a great deal. Multi-year ice is regarded as rigid, but lead ice is treated as an elastic beam (plate) on an elastic foundation and it breaks when the maximum bending moment reaches yield. The individual floes are rigid bodies subject to Newtonian mechanics. They interact under mutual collision and can also break and (owing to the energy provided by the lead ice pushed against the multi-year ice) pile up to form ridges. Hopkins and Hibler also calculate the ratio of the overall work to that of the potential energy of the ridge and find that it is about a factor of five larger than for ridges grown from a rubble-filled lead. The authors caution the reader from far-reaching inferences since computations are preliminary.

Work in a similar spirit is Shen and Ackley's onedimensional wave-induced ice-floe collision model for pancake ice herding together under wave action (Fig. 2e). The floes are treated as rigid bodies sliding along the free surface under the action of gravity. The frequency of collision is studied as a function of wave amplitude and the viscous and elastic properties of forces arising when two floes collide. The model requires extension to two dimensions, since sideways dispersion of floes, certainly of significance, is ignored.

\section{ICE-PLATE DYNAMICS AND MECHANICS}

Representation on this topic is relatively scarce, but analyses are thought-provoking. Squire and Fox study the role of incoming waves in ice-edge dynamics by treating the ice as an elastic, Reissner- or Mindlin-type plate and the ocean as an inviscid fluid under potential flow. Formulae for the reflection $(R)$ and transmission $(T)$ of an incoming wave $(I)$ are derived as functions of frequency, a)

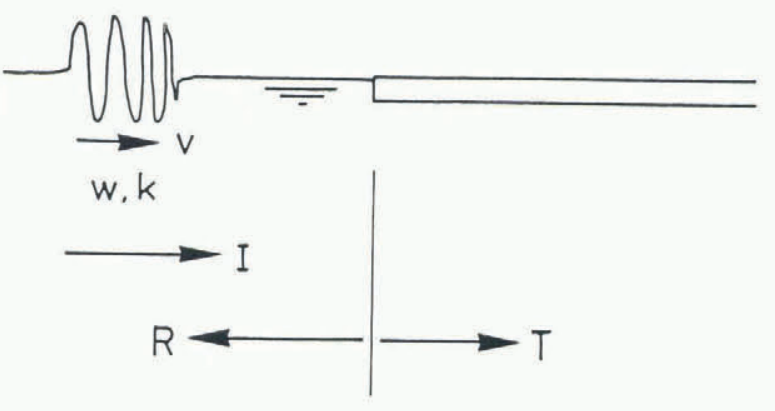

b) Erebus ice tongue

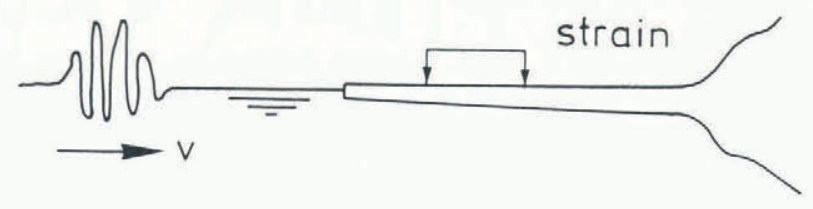

c)

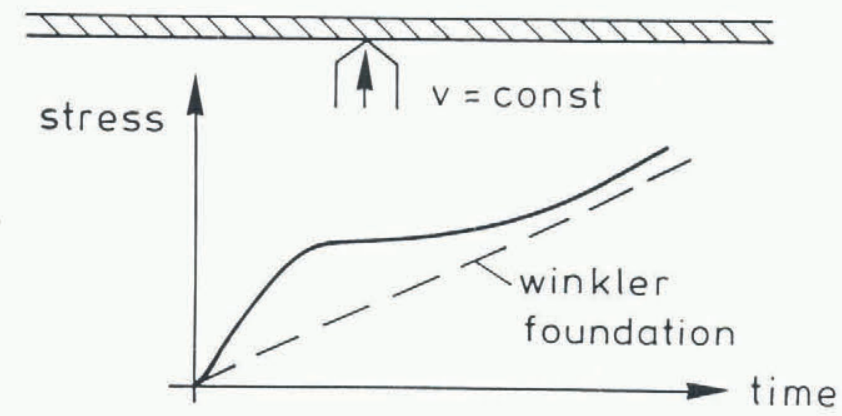

Fig. 3. (a) Reflection ( $R$ ) and transmission (T) of incoming (I) ocean waves at the edge of an elastic ice plate. (b) Interaction of ocean waves with an ice tongue on which time series of strains with 40-50 s periods were observed. (c) Indentation experiment of a floating ice plate from below with constant velocity. Stress versus time curves as measured (solid) and as obtained from a Winkler foundation hypothesis (dashed) (schematic).

$\omega$, and wavenumber, $k$, of the incoming wave (Fig. 3a). It is shown, by using Greenland Sea spectra, that the radiation stress at the ice edge due to the incoming waves is comparable in magnitude to, and sometimes larger than, the other forces that make up the momentum balance of an ice plate. The implications are that, both in sea-ice dynamics and ice engineering of floating plates, radiation stress ought to be included in a proper analysis. It would imply that ocean-wave spectra and intensities be known. Today, the effects may be "swallowed" in the numerical values of the constitutive constants.

An analysis in the same spirit is Fox and Squire's study of travelling waves in the Erebus Ice Tongue (Fig. $3 b)$. Very small measured surface strains, at periods between 40 and $50 \mathrm{~s}$, could be explained by forced waves transmitted beneath and coupled to the ice tongue. The transmission seems to be optimized at the right frequen- 
cies, but ocean waves at such long periods are weak. Reliable measurements, as suggested by participants, are likely to come from land-based pressure gauges or limnigraphs with appropriate filter devices. Boreholes drilled through the ice and recording water levels may be most appropriate because they will naturally filter out all insignificant periods of oscillation. Alternatively, the 40 to $50 \mathrm{~s}$ periodic response could be a standing wave of the Erebus Ice Tongue; such waves do also single out a particular frequency band of the incoming waves, but are always excited in an unsteady wave field.

A floating ice-plate analysis of engineering relevance is presented in the work by Dempsey and Zhao. They study the response of an infinite elastic plate on water of constant depth to an upward moving indentor, as sketched in Figure 3c, and prove that early time stresses obtained quasi-statically (with a Winkler foundation assumption) are considerably underestimated in comparison to those obtained when inertial forces of the fluid, but not of the plate, are incorporated.

\section{LOCAL ICE-OCEAN INTERACTION PHENOMENA, RELICT FLOW STRIPES}

There are only two papers dealing with localized phenomena. Pelto and Warren collect calving data for 20 tidewater glaciers and conjecture a linear relation between the height of the calving front and the calving velocity. They claim that Brown and others (1982), who came up with a similar functional relationship, overestimate the calving rate, but the scatter of the data from the 20 tidewater glaciers is so large that a dependence of the calving velocity on variables in excess of the front height is likely.

Alley studies the effects of sedimentation on oceanglacier interactions and focuses on the question of whether grounding-line deposits show a climatological imprint or not. According to his analysis, they may or may not, but the study nevertheless provides interesting information on the role of grounding-line advance and retreat.

Somewhat unrelated to any of the title topics of this review, but nevertheless studying local features, is Jeffries' paper on ancient sea-ice strata in the Ward Hunt Ice Shelf. By analyzing the physical structural characteristics of a $32 \mathrm{~m}$-deep ice core it is concluded that the sea ice has congelation structure, grown by Stefan accretion mechanisms to thicknesses far beyond the equilibrium thickness of multi-year ice, that it is permanent for up to several thousands of years and thus not affected by slow-acting physical processes.

Only marginal ice-ocean interaction is evident in a paper by Casassa and others, in which satellite imagery is used to interpret "Relict flow stripes on the Ross Ice Shelf". Advanced Very High Resolution Radiometer (AVHRR) data show that stripes, reminiscent of flowlines, are present over large portions of the Ross Ice Shelf. A large, looping pattern of such stripes west of Crary Ice Rise indicates, and present-day flowline patterns support, the conclusion that the stripes must represent relict flowlines that were generated approximately 800 years ago. The mechanism that produced these stripes remains largely a mystery, but the authors suggest that a raft of inland ice may have been torn from the grounded ice sheet about $400 \mathrm{~km}$ upstream of its present position. The implications call for caution in the interpretation of stripe features from satellite imagery as they may not represent flowline patterns of present ice flow.

\section{ATMOSPHERE-ICE-OCEAN INTERACTIONS}

Several levels of complexity are possible; some authors place their emphasis on the dynamics and treat momentum considerations carefully while somewhat glossing over energy budgets, others concentrate on careful energy balances and may omit momentum considerations altogether. Alternatively, the complexity of the interactions may be reduced by only looking at an incomplete coupling and, for example, using the atmosphere to drive a coupled ice-ocean system. On the other hand, all three systems could interact dynamically, yielding a full general circulation model (GCM). There are papers on almost all of these variants.

Ikeda, in his analysis of mesoscale eddy formation and evolution in the ice-covered ocean, studies the ocean only and treats the ice as drifting elements. The mesoscale eddies and currents, seen in NOAA images over the southern Labrador and Newfoundland shelves, are said to be due to barotropic, baroclinic, basically alongshore currents which, because of variations in bottom topography, are prone to instabilities. The model is quasi-geostrophic eddy-resolving and the geometry consists of a three-layer rectangular area with a two-layer shelf zone and a threelayer offshore portion. Two seamounts and a depression of rotational symmetry and Gaussian depth distribution are built in (see the author's Fig. 2). It is shown that eddies do indeed form as a result of barotropic-baroclinic instabilities. Anticyclonic and cyclonic eddies form at the upstream and downstream sides of the seamounts and the depression generates the opposite eddies. Furthermore, an alongshore, steady wind transforms a circular distribution of ice floes into an arch-shaped assembly; the deformation is due to drag variation of the wind over the open ocean and the ice cover, which generates Ekman divergence and thus the deformation. Coriolis effects also contribute to a drift towards the right (see the author's Figs. 3, 4 and 5).

McPhee presents a semi-analytic model for steady vertical momentum, heat and salt transport in the underice boundary layer (Fig. 4). Momentum balance (Fig. 4a) is treated by steady-state Ekman dynamics, with buoyancy taken into account. The eddy viscosity is assumed to vary linearly in a near-surface layer and is constant in the mixed layer above the pycnocline and a second constant below it (the values of which depend on the mixed-layer depth and eddy size, the buoyancy jump at the pycnocline and the buoyancy (Brunt-Väisälä) frequency). Heat and mass flux (Fig. 4b) account for icegrowth/melting rate at the ice-water interface, percolation of surface meltwater, heat and salt diffusion through a very thin laminar sub-ice layer followed by a turbulent heat and salt diffusion through the boundary layer. Physically important inferences of the model calculations are (1) that most change in temperature and salinity occurs within the laminar sub-layer and (2) that the drag 
a)

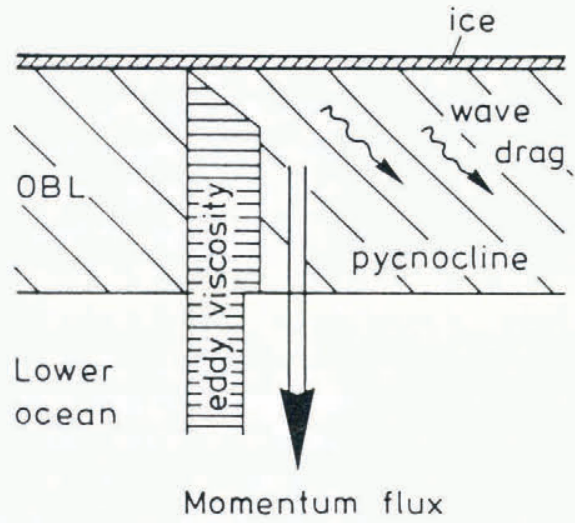

b)
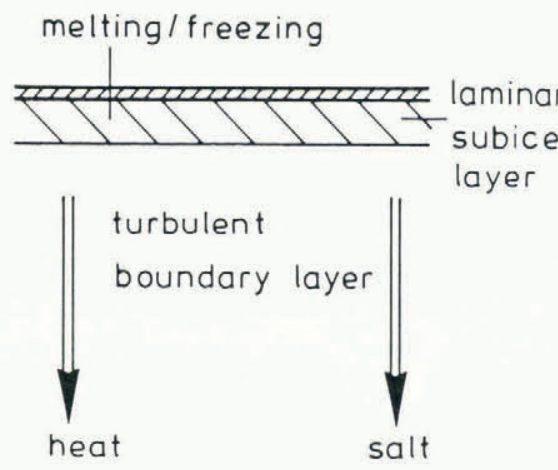

transport

Fig. 4. Explaining McPhee's quasi-analytic under-ice boundary-layer model. (a) Vertical momentum flux is treated by a steady Ekman balance in the ocean boundary layer (OBL) and the lower ocean below the pycnocline, with eddy viscosity distribution as sketched and drag due to internal waves being accounted for. (b) Heat and salt transport, which is affected by a laminar sub-layer and the ocean turbulent boundary layer, takes melting processes into account.

exerted by the ice on the water must include a component due to internal waves, the reason being that the strong stratification due to melting paired with the ice drift generates sufficient internal waves to make this contribution significant.

Work by Hibler and associates treats the ice-ocean in a dynamic-thermodynamic fashion, but the atmosphere does not participate fully as the driving agent in the interaction (Flato and Hibler, Ip and others, Zhang and Hibler, Hibler and Ranelli and WarnVarnas and others, all mentioned previously). In the same vein is work by Ries and Hibler, who construct an ice-ocean model and integrate it prognostically for the Arctic Ocean over three years with daily atmospheric forcing. The model has an $80 \mathrm{~km}$ grid resolution (as opposed to $160 \mathrm{~km}$ of the previous ones). The inferences from the analysis are that viscosities can be reduced and the topography better resolved, so that the baroclinic motion is better predicted, but refinement does not lead to improved ice-edge prediction, thus model improvements (but which ones?) may be needed.

A study involving energy considerations of mesoscale significance is Prinsenberg and others' paper on "Estimates of ice-edge melt rates along the Canadian east coast". The melt rates are estimated from ice-edge thickness records and satellite-tracked ice beacons and ice charts which permit determination of drift and ice-edge velocities. With these data, the required heat to melt the ice can be estimated for each beacon. It is found that the melt rates and associated heat fluxes required to melt the ice depend on the relative movement of the ice to the ocean and on the ocean temperature. Over the continental slope, the ice-edge melt rates are considerably larger than on the continental shelf, the reasons being that the wind causes upwelling and shoreward advection of warm water along the slope area. The data of all beacons permit a linear regression fit between (1) the heat fluxes required to melt the ice around the beacons and normalized ice melt rates and (2) the ice-edge drift rates and their components perpendicular to the ice-edge boundary.

Observational work in a similar spirit is reported by Muench and others in their paper on oceanic and meteorological effects on autumn sea-ice distribution in the Bering and Chukchi seas during fall 1987 and 1988. Iceedge locations and ice-drift information indicate that the position of the ice edge in 1988 was considerably farther south than in other years and that this was due to strong northerly and northwesterly winds which held the summer pack ice trapped against the beach. The meltwater from this ice stratified the upper water columns that the ice became effectively insulated against vertical flux of heat from the underlying warm water.

Another mesoscale energy study is Steffen's "Energy flux density estimation over sea ice in the North Water region". The analysis is based on passive microwave measurements of the microwave radiometer aboard the Nimbus 7 satellite. Since the polarization ratio (18 and $37 \mathrm{GHz}$ ) is increasing for decreasing ice thickness, it can be used to classify different ice types and these, in turn, provide a basis for the estimation of the energy-flux density through the ice. With this method, it is found that the mean energy loss for the entire North Water region is $77 \mathrm{~W} \mathrm{~m}^{-2}$ for the months of November to March, of which about half is due to refreezing of ocean water and half due to withdrawal from the enthalpy.

Work in which momentum balance is ignored entirely and the energy balance is essentially treated in a parameterized form is Worby and Allison's paper on "Ocean-atmosphere energy exchange over thin, variableconcentration Antarctic pack-ice". The starting point is the recognition that the average flux of sensible and latent heat through sea ice does not only depend on the areal concentration of lead ice, thin ice and thick ice 
but reacts, in addition, in a non-linear fashion to the lead width. The ocean-atmosphere energy exchange is studied by using Maykut's 1978 thin-ice energy-budget model and combining it with an empirical parameterization for the turbulent fluxes from leads. The model computations are performed for a constant floe size, and ice concentrations are varied by changing the width of the leads between floes.

The computational results indicate that at $80 \%$ areal ice concentration the turbulent heat loss can be greater than that from open water but, as concentration decreases, the fractional heat loss through the ice becomes less and is practically negligible when ice concentration is less than $50 \%$. Unfortunately, owing to lack of data, no observational verification of the results is given.

A coupled sea-ice-oceanic mixed-layer model for the Southern Ocean that is forced by daily atmospheric variables as presented by Stössel, is run (1) by specifying the surface boundary conditions and (2) by adding an additional coupled one-dimensional atmospheric boundary layer. The results show that the model predictions in (2) suffer from a poor prediction of the ice extent, thicknesses and velocities. Adjustments in the original model parameters and physics are discussed that provide improvement of the prediction of these quantities.

A fully coupled ocean-sea-ice-atmosphere model, that includes interaction and feedback, is the dynamic sea-ice model for the Antarctic for use with an atmospheric GCM by Budd and others. The ocean consists of a fully-mixed dynamic upper layer and a deep ocean. The ice model permits ice concentration and thickness to be prognosticized, its rheological properties being those of an incompressible cavitiating fluid (see Ip and Hibler and Fig. 2), complemented by an ad hoc compression rheology. Both are coupled to an atmospheric model that computes heat fluxes, sea-ice growth and changes in concentration. Preliminary sensitivity studies show reasonable and stable simulations for the present mean Antarctic winter climate.

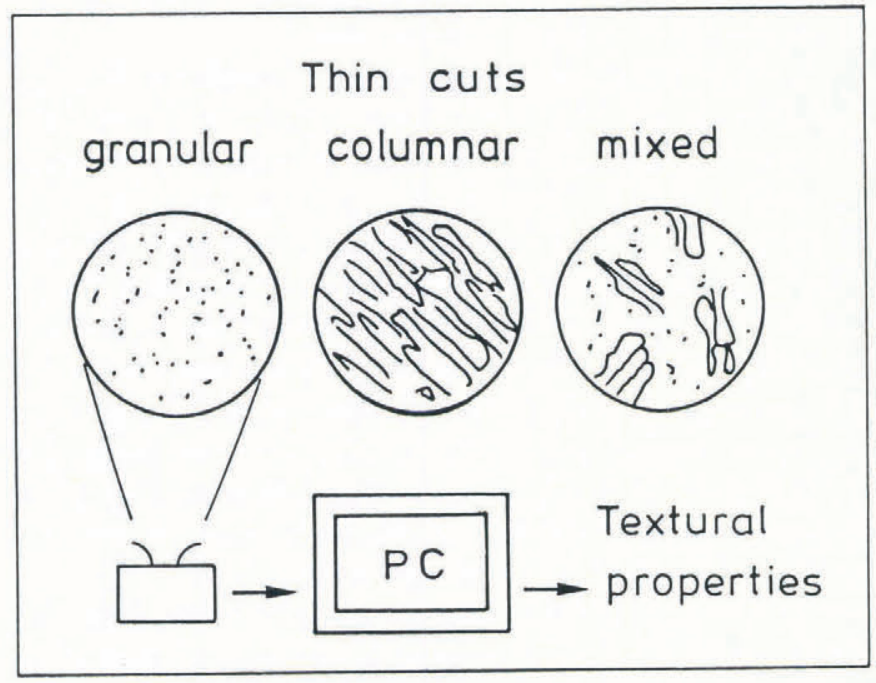

Fig. 5. Thin cuts under polarized light are photographed, the photos digitized and analyzed in a PC for two textural parameters, "constant gradient" and "cord-size distribution" of grains.

\section{MECHANICS AND MATERIAL SCIENCES}

Contributions can be divided into analyses of in situ measurements and laboratory studies.

\section{Laboratory studies}

It has long been known that the textural properties of ice may affect its mechanical properties, but the subjective character of the traditional visual classification hinders comparison between large numbers of samples and prohibits a high degree of reproducibility. Eicken and Lange report on the development of an imageprocessing technique that permits them to differentiate between granular, columnar and mixed-type sea ice (Fig. 5). Thin sections of sea ice between two polarizers are photographed and then pixel-processed on a PC for contrast properties. Two parameters, the "contrast gradient" between grains and the skewness of the cord-size distribution (the cord size is the length of the perimeter of a grain) are shown to suffice in order to distinguish reliably between granular, columnar and mixed sea-ice textures.

Unfortunately, this method does not seem to be used systematically by Lange and Eicken in their second paper on the textural characteristics of sea ice and the major mechanisms of ice growth in the Weddell Sea. They find that, by and large, Antarctic sea ice is dominated by granular ice of frazil origin in floes of all ages, in contrast to ice in the Arctic, the reason most likely being the larger deformational activity in the Southern Ocean due to the strong circumpolar current.

A series of experimental studies on the material response of fresh water and sea ice includes Schulson and others' analysis of the effect of confinement on the brittle compressive fracture of coarse-grained polycrystalline fresh-water ice. Cubes of $150 \mathrm{~mm}$ side-length are compressed under isotropic confinement $\left(\sigma_{2}=\sigma_{3}\right)$ and for a range $0<R<0.3$ of the principal stress ratio $R=\sigma_{2} / \sigma_{1}$ (Fig. 6a). It is found that the compressive stress, $\sigma_{1}$, at failure depends on the confinement ratio $R$. Two regimes can be identified: for $R \leq 0.15$, failure stress is strongly a)

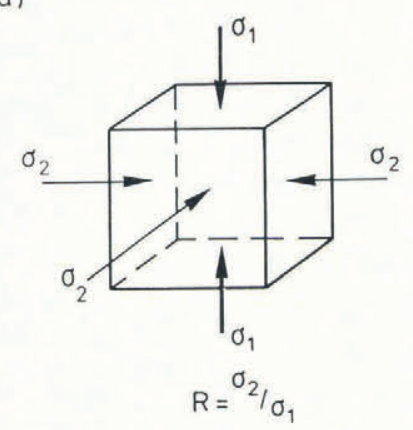

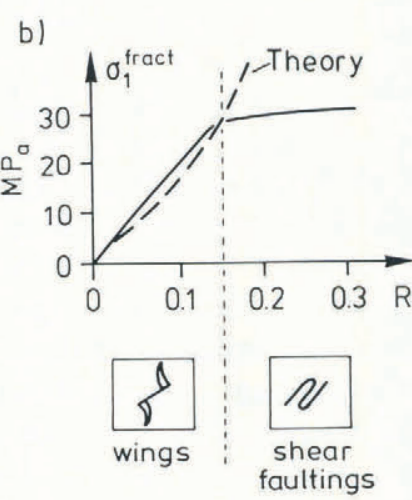

Fig. 6. Compressive fracture under confinement. (a) Stress state on cube with $150 \mathrm{~mm}$ side-length. (b) Fracture stress, $\sigma_{1}^{\text {fract }}$, as a function of confinement ratio, $R$, and sketches of fracture modes in the two regimes for $R$ where behaviour is different. 
dependent on $\mathrm{R}\left(\mathrm{d} \sigma_{1} / \mathrm{d} R \sim 175 \mathrm{MPa}\right)$; for higher values, $0.15<R<0.3$, this dependence is substantially weaker ( $\mathrm{d} \sigma_{1} / \mathrm{d} R \sim 17 \mathrm{MPa}$ ) (Fig. $6 \mathrm{~b}$ ). The two failure modes are accompanied by different failure mechanisms: at low confinement, axial splitting with wing crack formation is predominant (Fig. 6b); at higher confinement, shear faulting as well as splitting occurs. The former is explained with a theoretical model, the latter is not.

An obvious extension of this work is the determination of principal failure stress under anistropic confinement. On the other hand, the two types of failure behaviour are likely to be influenced by crack nucleation. For uniaxial compression $(R=0)$, results of tests on granular fresh-water ice are reported by Kalifa and others. They study the process of crack nucleation at strain rates of the order $10^{-3} \mathrm{~s}^{-1}$, grain-sizes of 1 to $10 \mathrm{~mm}$ and temperatures between -2 and $-40^{\circ} \mathrm{C}$. They observe formation of the first crack in their specimen and record the relaxation properties upon load removal immediately after crack formation. The results are interpreted in terms of grain boundary sliding, controlled by the intrinsic viscosity of the boundary. It would be interesting to see how these results were changed when sideways confinement is introduced.

It is intuitively obvious that propagation of a crack under load will depend upon the sharpness of the crack tip. Thus, fracture toughness (the resistance of a crack to propagate under loading) will depend on notch acuity (crack tip sharpness). This has been studied recently for fresh-water ice, and the same authors (De Franco and others) describe results for sea ice. By fabricating notches in four different ways, specimens with four different crack tip radii, $\rho$, are obtained for which the fracture toughness can be determined. It is found that fracture toughness grows with the tip radius. For sharper crack tips $(\rho \leq 1 \mathrm{~mm})$, this dependence is very weak; for $\rho \geq 1 \mathrm{~mm}$ it is significant even though scatter of the preliminary data is large (Fig. 7a).

Notch acuity will undoubtedly affect the fatigue strength of ice under cyclic loading, since crack growth depends on it. This is so for both fresh-water ice and sea ice. The study of the former for S2 ice (i.e. columnar ground ice with the $c$ axis in the horizontal plane) is presented by Nixon and Weber. It is demonstrated that crack growth is indeed enhanced by cyclic loading.

The kinetic friction coefficient $\mu$ (i.e. the ratio of the tangential to the normal force) of laboratory-grown saline ice against itself under varying conditions of velocity and ambient temperature is studied by Jones and others. For some temperatures they find that $\mu$ may first grow with sliding velocity, reach a maximum and subsequently fall again. For other temperatures, $\mu$ is monotonically decreasing with sliding velocity (Fig. 7b). These results clearly indicate that the proper functional dependence has not yet been captured and a dimensional analysis of the problem is suggested.

Two papers deal with indentation experiments on beams. Sodhi monitors the force, and the work used up and that lost, when the beam against which the indentor presses crunches. This work is obviously of relevance in off-shore technology. On the other hand, Gagnon and Mølgaard give experimental evidence that seems to prove that the high pressure that occurs between the indentor plate and the ice gives rise to regelation processes, i.e. water formation during loading and refreezing under unloading. They show that at least $50 \%$ of the energy during the process of indentation is consumed by pressure-melting and heat generation through the rapid viscous flow of the liquid.

\section{In situ measurements}

Jacka and others report on an Antarctic field study of the movement and rheology of a sea-ice floe. A strain grid consisting of six canes, placed around a ship that was beset in the ice, is established. The distances to each cane and the horizontal angles between them and nearby Mt Biscoe (Antarctica) are measured daily. These measurement allow estimation of the ice-floe deformation field and provide hints as to the appropriateness of the assumed sea-ice rheology that is used in numerical cli-

\section{a)}

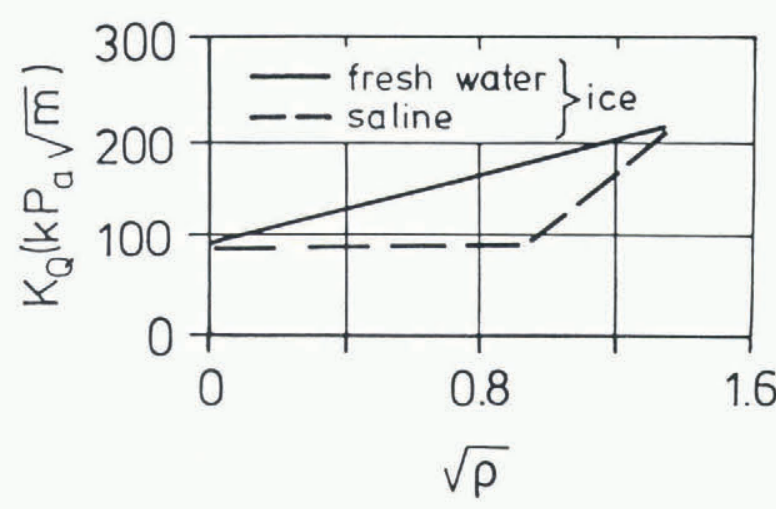

b)

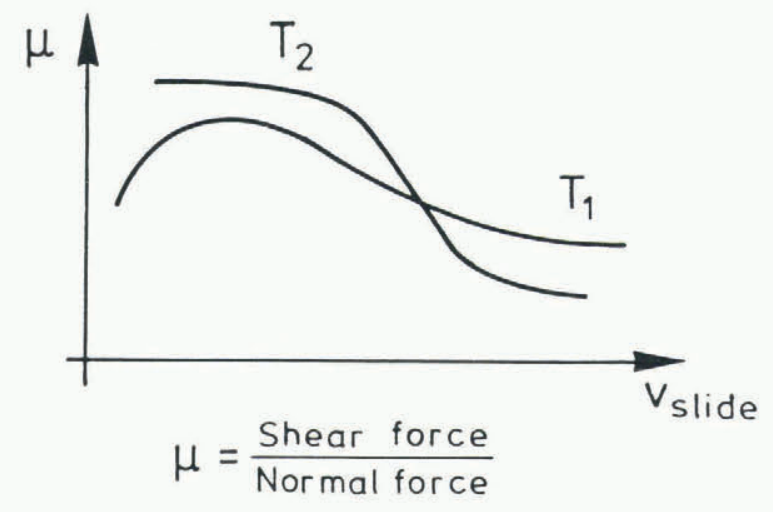

Fig. 7. (a) Fracture toughness, $K_{Q}$, plotted against crack tip radius, $\rho$, for fresh-water and saline ice as inferred from DeFranco and others. (b) Kinetic friction coefficient, $\mu$, of laboratory-grown saline ice against itself as a function of the sliding velocity and various temperatures (schematic). 
mate modelling. True, the measurements are very crude, but Budd mentioned in the discussion that these are the most valuable measurements taken recently, because they are the first ones being performed in situ that provide any clue as to the correctness of postulates regarding sea-ice rheology.

A similar set of field measurements, though performed differently, is described by Tucker and others. From an ice-strengthened ship, moored to a multi-year floe in the pack ice northeast of Svalbard, stress sensors were placed at four sites around the ship, each consisting of a rosette permitting principal stress and principal strain direction to be determined. Furthermore, microwave transponders, capable of measuring deformation, are positioned around the area. The distances from the ship to the transponders is much larger than to the stress sensors. Spectral analyses of the recorded time series indicate that large-amplitude long-period events are well correlated between the sites whereas small-amplitude highfrequency episodes are not. Data suggest that the seaice cover exhibits, in parts, bending deformation with a conspicuous $12 \mathrm{~h}$ oscillation. The measurements are interesting because they embrace large ridging events and the break-up of the multi-year floe into small fragments.
Sayed and Frederking examine ice-surface geometry at the shear edge of landfast ice. From an areal survey, they obtain surface profiles and evaluate the height statistics. They find that, at a shear edge, the height follows a log-normal distribution whereas in areas inside the shear edge it follows a negative exponential distribution.

\section{ACKNOWLEDGEMENTS}

In carrying out this work, I was supported in part by the Geographical Institute, ETH, Zürich. I thank Mrs Danner for typing the text of this summary.

\section{REFERENCE}

Brown, C.S., M.F. Meier, and A. Post. 1982. Calving speed of Alaska tidewater glaciers with applications to the Columbia Glacier, Alaska. U.S Geol. Surv. Prof. Pap. 1258-C.

The accuracy of references in the text and in this list is the responsibility of the author, to whom queries should be addressed. 